\title{
Ulceration of the oral mucosa induced by antidepressant medication: a case report
}

\author{
Fernanda Bertini ${ }^{1}$, Nívea Cristina Sena Costa ${ }^{1}$, Adriana Aigotti \\ Haberbeck Brandão², Ana Sueli Rodrigues Cavalcante ${ }^{3}$ and \\ Janete Dias Almeida*3
}

\begin{abstract}
Address: ${ }^{1}$ Postgraduate Program in Biopathology and Department of Biosciences and Oral Diagnosis, São José dos Campos Dental School, São José dos Campos, São Paulo, Brazil, ²Discipline of General Pathology, Department of Biosciences and Oral Diagnosis, São José dos Campos Dental School, São José dos Campos, São Paulo, Brazil and ${ }^{3}$ Discipline of Stomatology, Department of Biosciences and Oral Diagnosis, São José dos Campos Dental School, São José dos Campos, São Paulo, Brazil

Email: Fernanda Bertini - bertinife@uol.com.br; Nívea Cristina Sena Costa - niveasena@yahoo.com.br; Adriana Aigotti Haberbeck Brandão - aigotti@fosjc.unesp.br; Ana Sueli Rodrigues Cavalcante - sueli@fosjc.unesp.br; Janete Dias Almeida* - janete@fosjc.unesp.br

* Corresponding author
\end{abstract}

Published: 3 November 2009

Journal of Medical Case Reports 2009, 3:98 doi:10.1186/1752-1947-3-98
Received: 30 April 2008

Accepted: 3 November 2009

This article is available from: http://www.jmedicalcasereports.com/content/3/1/98

(C) 2009 Bertini et al; licensee BioMed Central Ltd.

This is an Open Access article distributed under the terms of the Creative Commons Attribution License (http://creativecommons.org/licenses/by/2.0), which permits unrestricted use, distribution, and reproduction in any medium, provided the original work is properly cited.

\begin{abstract}
Introduction: Ulcers are frequent lesions of the oral mucosa. Generally, they are circumscribed round or elliptical lesions surrounded by an erythematous halo and covered with an inflammatory exudate in their central portion, and are accompanied by painful symptoms. Oral ulcers affect $20 \%$ of the population, especially adolescents and young adults. The etiopathogenesis includes immunological alterations, infections, nutritional deficiency, trauma, food and contact allergies, autoimmune diseases, neoplasms, and psychosomatic, genetic and environmental factors.

Case presentation: A 78-year-old Caucasian woman was referred by her dentist to our outpatient clinic with a 4-week history of an oral ulceration after using an antidepressant (sertraline hydrochloride). On the basis of the clinical findings and anamnesis, the occurrence of the lesion was attributed to the use of the drug. Exfoliative cytology was performed, to reassure the patient that it was not oral cancer, which revealed the presence of a nonspecific inflammatory reaction. The drug was replaced and resolution of symptoms was observed.

Conclusion: Exfoliative cytology should be the complementary examination of choice in cases of oral ulcers with a suspicion of drug interaction. Although this is a rare event in dental practice, dentists should be aware of the diagnostic possibility of drug-induced ulcers and should cooperate with the clinician to adjust the prescribed medication to resolve the symptoms.
\end{abstract}

\section{Introduction}

Oral ulcers are inflammatory lesions of the oral mucosa that affect approximately $20 \%$ of the population [1]. These ulcers are accompanied by painful symptoms and are generally characterized by shallow oval circumscribed lesions surrounded by an erythematous halo and covered with a fibrinous exudate in their central portion. Oral 
ulcers manifest as acute (duration of up to 6 weeks), chronic, or recurrent solitary or multiple lesions [2].

Numerous causes and factors involved in the formation of these lesions have been reported in the literature, including immunological alterations, infections, nutritional deficiency, repetitive trauma to the mucosa, food and contact allergies, autoimmune diseases and neoplasms, as well as psychosomatic, genetic and environmental factors [3].

Sertraline hydrochloride is an antidepressant agent used in clinical practice, which acts on the serotonin neurotransmitter. Antidepressants such as selective and potent serotonin reuptake inhibitors potentiate serotonergic neurotransmission and show a low frequency of adverse reactions [4].

A variety of cases of ulcerated lesions have been reported in the oral mucosa associated with the use of different systemic medications. This is of great interest to dentists who are frequently responsible for the diagnosis of these lesions. We report the case of a patient who developed oral ulceration after the use of an antidepressant (sertraline hydrochloride).

\section{Case presentation}

A 78-year-old Caucasian woman was referred by her dentist to our outpatient clinic, with a 4-week history of an oral ulceration. She mentioned that she had basocellular carcinoma in her face and stomach cancer. Extra-oral clinical examination showed facial symmetry and palpable, mobile, smooth and asymptomatic submandibular lymph nodes. A shallow ulcer with an erythematous border, measuring about $1.5 \mathrm{~cm}$ at its maximum diameter, was noted upon intraoral examination. The ulcer was located in the mucosa of the lingual region of the left lower premolars (Figure 1) and was accompanied by painful symptoms.

Upon anamnesis, the patient reported the use of sertraline hydrochloride for the treatment of depression at an initial dose of $50 \mathrm{mg}$, which was subsequently increased to 100 $\mathrm{mg}$ when the occurrence of the lesion was first noted. After evaluation of the medication by the physician responsible, sertraline was replaced by $75 \mathrm{mg}$ of venlafaxine and exfoliative cytology of the ulcer was performed.

Exfoliative cytology of the lesion showed superficial and intermediate pavement epithelial cells presenting diverse inflammatory and degenerative alterations, such as vacuolization, a perinuclear halo, an enlarged nucleus, binucleation and lysis, numerous mono- and polymorphonuclear leukocytes, thick and filamentous

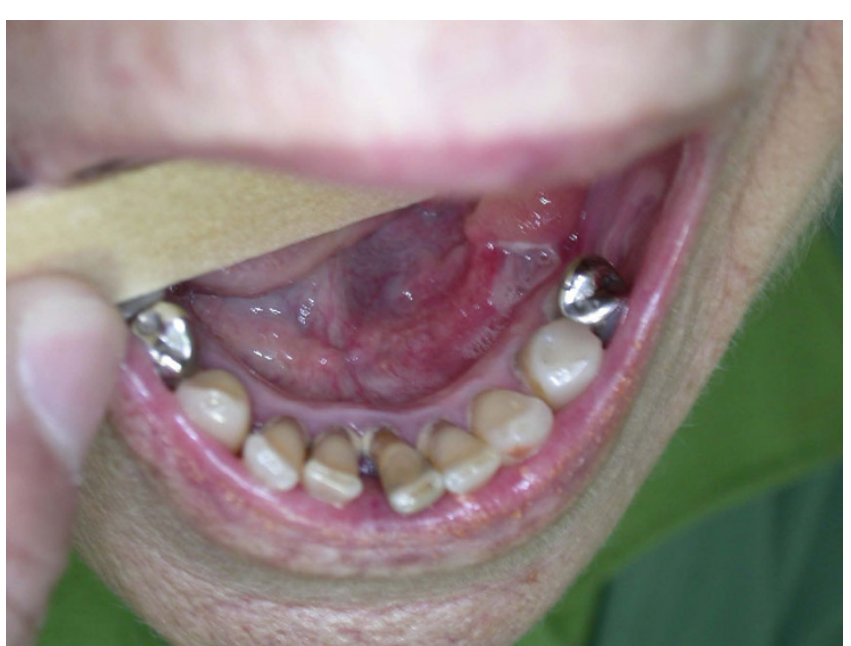

Figure I

Shallow ulcer with an erythematous border, measuring about $1.5 \mathrm{~cm}$ at its maximum diameter, located in the mucosa of the lingual region of the left lower premolars.

mucus, and cell remnants, in addition to a mixed flora consisting of bacteria and Candida hyphae (Figure 2).

Mouth rinsing with a betamethasone elixir three times per day for 3 minutes was initially prescribed for a period of 5 days. On her return visit, the patient presented with improvement of the clinical symptoms and was instructed to continue mouth rinsing for an additional 7 days. Two weeks later, she returned, complaining of persistent pain in the affected area. The patient returned after 21 days showing significant improvement of the clinical symptoms and re-epithelization of the ulcer. The patient was followed up for 4 months at weekly intervals and was discharged after this period. She presented with no further oral complaints over 2 years of follow-up.

\section{Discussion}

Our patient presented with an oral ulcer and was referred by her dentist, who was worried about the possibility of oral cancer. Oral ulcers can be the first manifestation of systemic diseases of immunogenetic origin, such as Behçet's disease [5] and others. Diseases such as pemphigus and pemphigoid may also impair differential diagnosis with nonspecific secondary ulcers after rupture of the bullae [6]. In such cases, a biopsy combined with immunofluorescence is a fundamental tool for a definitive diagnosis.

Deficiencies in iron, folic acid, $\mathrm{B}_{12}$ complex and $\mathrm{B}_{6}$ vitamins, and trace elements such as zinc have been variably related to the occurrence of ulcers [7]. 


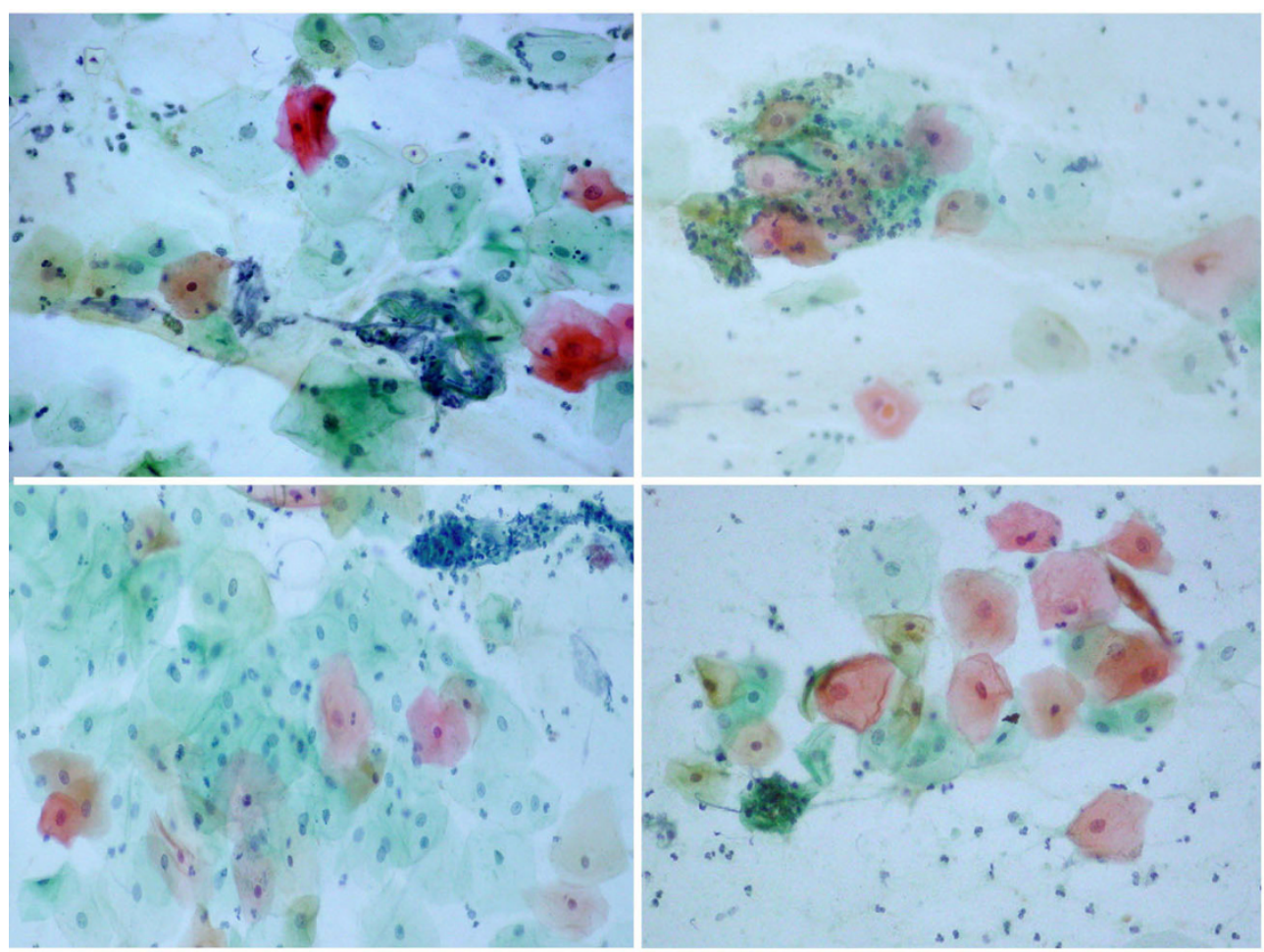

\section{Figure 2}

Smears showing superficial and intermediate epithelial cells with inflammatory and degenerative alterations such as a perinuclear halo, cytoplasmic lysis and vacuolization, and numerous leukocytes, sometimes aggregated with filamentous mucus (Papanicolaou stain, 630x).

Several studies have evaluated the use of drugs that may induce the formation of ulcers, including medications such as niflumic acid, captopril, piroxicam and phenobarbital. Cytotoxic agents used in antineoplastic therapies affect dividing cells, a phenomenon that manifests in the oral mucosa, inducing ulcers in some patients [8]. Alendronate, a drug prescribed for the treatment of patients with osteoporosis, has also been associated with the occurrence of oral ulcers. In a review of 200 patients investigating the adverse effects of drugs in the oral cavity, Smith and Burtner observed dry mouth in $80.5 \%$, dysgeusia in $47.5 \%$ and stomatitis in $33.9 \%$ [9]. In another review on the adverse effects of drugs, Scully and Bagan reported adverse manifestations such as hypersalivation, white lesions, burning mouth sensation, mucositis, neoplasms, pemphigus, pemphigoid and other bullous disorders, mucosal pigmentation, lichenoid reactions, cheilitis, neuropathies, and halitosis [10]. The authors reported that aphthous ulcerations were observed after the use of $\beta$-blockers such as labetalol, captopril, nicorandil and nonsteroidal antiinflammatory drugs and also after the use of mycophenolate or sirolimus, sodium lauryl sulfate, protease inhibitors, and sulfonamides.
In the era of transplantation, a frequent medical concern is the development of ulcers that might be exacerbated by the administration of immunosuppressive medications such as mycophenolate mofetil, which has been used in combination with calcineurin inhibitors and steroids. The drug-induced ulcerated lesions disappear when the medication is discontinued [11].

One study has shown the development of oral ulcers in four patients with angina pectoris who used nicorandil, a nicotinamide ester. In the cases studied, the lesions improved after dose reduction or interruption of the medication [12].

Stress has been identified as an important factor triggering the occurrence of oral ulcers, with the use of antidepressants being recommended in some patients for the control of recurrent aphthous ulcers [13]. However, in this study, after the clinical diagnosis of depression and prescription of sertraline hydrochloride, the patient developed an adverse reaction to the drug characterized by the occurrence of an ulcer in the oral mucosa. Replacement of the medication was requested, which led to the improvement of symptoms. 
As the patient had a previous history of stomach cancer, she was upset about the possibility of the ulcer being an oral cancer lesion. Exfoliative cytology was performed to lessen the patient's worries about the lesion. Exfoliative cytology is a viable alternative and should be the complementary examination of choice in these situations. Because an early diagnosis of oral cancer is essential for survival and to minimize public health expenses, exfoliative cytology might be used as a complementary examination in the monitoring of cancer risk factors [14].

Oral cancer mainly affects the floor of the mouth, the lateral border of the tongue and the soft palate, although other areas of the mouth may also be involved. Many cases of oral cancer are diagnosed during the advanced phase, a fact that results in an unfavorable prognosis and high mortality, in addition to high costs of treatment and an increased number of complications. Thus, an early diagnosis and preventive approach are of extreme importance in this disease [15].

The clinical manifestations of adverse reactions to drugs depend on the dose and type of medication, as well as on individual differences related to the patient. These reactions might be rapid or persist for a number of days after the use of the drug. According to the literature and to clinical practice, in most drug-induced reactions improvement of clinical symptoms occurs after dose reduction or interruption of the medication. Generally, these adverse reactions occur in the first or second week after the beginning of the therapy of choice and depend on the dose and cumulative toxicity of the drug, with the reactions usually ranging from moderate to severe. However, severe reactions require rapid withdrawal of the drug or its replacement. Many patients use multiple systemic medications that may eventually be supplemented with other drugs necessary for dental treatment [10].

Oral ulcers are frequent in oral diagnosis clinics and the lesions must be carefully examined, including the aspect of the surface, the presence of an erythematous halo and the deepness of the lesion. The etiological diagnosis is based on the presence of associated signs and symptoms that should be investigated during anamnesis.

The dentist should have pharmacological knowledge of the prescribed drug and of its possible interaction with other medications. Thus, cooperation between the treating doctor and the dentist is necessary in order to choose the best treatment that will guarantee the well-being and best quality of life of the patient.

\section{Conclusion}

Exfoliative cytology should be the complementary examination of choice in cases of oral ulcers with a suspicion of drug interaction. Although this is a rare event in dental practice, the dentist should be aware of the diagnostic possibility of drug-induced ulcers and should cooperate with the clinician to adjust the prescribed medication for symptom resolution.

\section{Consent}

Written informed consent was obtained from the patient for publication of this case report and any accompanying images. A copy of the written consent is available for review by the Editor-in-Chief of this journal.

\section{Competing interests}

The authors declare that they have no competing interests.

\section{Authors' contributions}

JDA and FB analyzed and interpreted the patient data regarding the clinical aspects. AAHB performed the cytological examination of the smears, NCSC and ASRC were contributors in writing the manuscript. All authors read and approved the final manuscript.

\section{References}

I. Scully C, Shotts R: ABC of oral health. Mouth ulcers and other causes of orofacial soreness and pain. Br Med J 2000, 321:162-165.

2. Schneider LC, Schneider AE: Diagnosis of oral ulcers. Mt Sinai J Med 1998, 65:383-387.

3. O'Neill A, de Leon J: Two case reports of oral ulcers with lamotrigine several weeks after oxcarbazepine withdrawal. Bipolar Disorders 2007, 9:310-313.

4. Moreno RA, Moreno DH, Soares MBM: Psicofarmacologia de antidepressivos. Rev Bras Psiquiatr Depressão 1999, 21:24-40.

5. Areny R, Saavedra J: Enfermedad de Behcet: Experiencia en el Departamento de Reumatología. Hospital San Juan de Dios. Reumatologia 2003, 19:150-152.

6. Scully C, Challacombe S): Pemphigus vulgaris: update on etiopathogenesis, oral manifestations, and management. Crit Rev Oral Biol Med 2002, 13:397-408.

7. Jones TA, Parmar SC: Oral mucosal ulceration due to ferrous sulphate tablets: report of a case. Dent Update 2006, 33(10):632-633.

8. Scully C, Gorsky M, Lozada-Nur F: The diagnosis and management of recurrent aphthous stomatitis: a consensus approach. J Am Dent Assoc 2003, I34:200-207.

9. Smith RG, Burtner AP: Oral side-effects of the most frequently prescribed drugs. Spec Care Dent 1994, 14:96-102.

10. Scully C, Bagan JV: Adverse drug reactions in the orofacial region. Crit Rev Oral Biol Med 2004, I 5:22 I-239.

II. Naranjo J, Poniachik J, Cisco D, Contreras J, Oksenberg D, Valera JM, Díaz JC, Rojas J, Cardemil G, Mena S, Castillo J, Rencoret G, Godoy J, Escobar J, Rodríguez J, Leyton P, Fica A, Toledo C: Oral ulcers produced by mycophenolate mofetil in two liver transplant patients. Transplant Proc 2007, 39(3):6|2-6|4.

12. Healy CM, Smyth Y, Flint SR: Persistent nicorandil induced oral ulceration. Heart 2004, 90:37-38.

13. Pedersen A: Psychologic stress and recurrent aphthous ulceration. J Oral Pathol Med 1989, I 8: I 19-122.

14. Fontes PC, Corrêa GHM, Issa JS, Almeida JD: Quantitative analysis of AgNOR proteins in exfoliative cytology specimens of oral mucosa from smokers and non-smokers. Anal Quant Cytol Histol 2008, 30(I):16-24.

15. Fontes PC, Corrêa GHM, Issa JS, Almeida JD: Comparison of exfoliative Pap stain and AgNOR counts of the tongue in smokers and nonsmokers. Head Neck 2008, 2(3):157-162. 